\title{
A New Formulation for the Combined Maritime Fleet Deployment and Inventory Management Problem
}

\author{
Bo Dong ${ }^{1}(\otimes)$, Tolga Bektaş ${ }^{2}$, Saurabh Chandra ${ }^{3}$, Marielle Christiansen ${ }^{1}$, and \\ Kjetil Fagerholt ${ }^{1,4}$ \\ 1 Norwegian University of Science and Technology, Trondheim, Norway \\ bo.dong, mc,kjetil.fagerholt@ntnu.no \\ ${ }^{2}$ University of Southampton, Southampton, United Kingdom \\ T.Bektas@soton.ac.uk \\ 3 Indian Institute of Management Indore, P Madhya Pradesh, India \\ saurabh@iimidr.ac.in \\ ${ }^{4}$ SINTEF Ocean, Trondheim, Norway
}

\begin{abstract}
This paper addresses the fleet deployment problem and in particular the treatment of inventory in the maritime case. A new model based on time-continuous formulation for the combined maritime fleet deployment and inventory management problem in Roll-on Roll-off shipping is presented. Tests based on realistic data from the Ro-Ro business show that the model yields good solutions to the combined problem within reasonable time.
\end{abstract}

Keywords: Fleet deployment, Maritime inventory routing, Ro-Ro shipping

\section{Introduction}

In maritime transportation, ships typically operate in one of the three modes: industrial, tramp or liner shipping. In industrial shipping, a shipping company manages the ships and the cargoes to be transported, with the aim of minimizing the transportation costs. In tramp shipping, the ships are assigned to the cargoes (some of which may not be obligatory) under contracts between the shipping company and the cargo owners, with the aim of maximizing profits, similar to a taxi service. In liner shipping, the ships follow a predefined itinerary with given port calls along routes according to a published schedule, similar to a bus service. Christiansen et al. [7] provide a detailed review on ship routing and scheduling for the various operational modes.

Roll-on Roll-off (Ro-Ro) shipping, a segment within liner shipping, is the major mode for the long distance international transportation of automobiles and other types of rolling equipment, as well as cargoes that can be placed on trolleys for loading and unloading. Meanwhile, the involvement of container shipping companies in automobile transportation business provide tough competition to Ro-Ro shipping companies. To improve profitability and strengthen 
the ties with its customers, some Ro-Ro shipping companies may consider to provide vertically integrated logistics services to automobile companies, where a Ro-Ro shipping company is responsible for inventory management at the ports and transportation at sea [5]. The automobile companies share the production and consumption information with the shipping companies, while the shipping companies share the ship schedules with automobile companies on a regular basis.

Fleet deployment can be considered as a tactical planning problem of assigning ships in the fleet to voyages that must be serviced regularly on given geographical routes. The fleet deployment gives which ship will perform which voyage, as well as sailing routes for each ship in the fleet, i.e. each ship is assigned a sequence of voyages to service, probably with ballast sailing between the last port of one voyage and the first port of the next voyage. In most literature, fleet deployment problems were first encountered in container shipping, the largest segment within liner shipping. The models for fleet deployment problems in container shipping usually assume:

1. Each ship is assigned a single route and loop during the planning horizon.

2. Individual ships of the same type are not distinguished.

Perakis and Jaramillo $[10,12]$ have contributed to develop mathematical models for liner shipping fleet deployment problems. They proposed a linear programming (LP) model for a liner ship fleet deployment problem. The LP model minimizes the total operating costs for a fleet of liner ships over a given planning horizon. The model assigns ships across the routes, determines the number, type and duration of chartering-in ships and owned ships that are laid-up during the planning horizon. In their approach, the ship speed is considered as a parameter in the LP model. Their work was extended by Powell and Perakis [13]. They proposed an integer programming (IP) formulation to optimally assign fleet of ships for a real liner shipping company. Computational results show substantial savings in total costs in comparison with the manual planning.

In Ro-Ro shipping, ship route planning works under more flexible assumptions. Fagerholt et al. [8] present a mixed integer programming (MIP) model for fleet deployment in Ro-Ro shipping, and Andersson et al. [3] extend this model for a real fleet deployment problem by including speed as a decision variable.

Inventory management deals with deciding the quantities to transport between the ports along the routes so that the port inventory level of products are kept within given limits. There are a few examples in the literature for maritime inventory routing problems, though mostly in the industrial and tramp shipping (see for example $[1,6,9,11,14-17]$ ). These literature suggest substantial financial savings by combining ship routing and scheduling planning with inventory management in industrial and tramp shipping. Therefore, it can be inferred that the fleet deployment planning in Ro-Ro shipping can achieve better results by integrating inventory management of cargoes at associated production and consumption ports.

In this paper, we propose a new time-continuous formulation for the combined Maritime Fleet Deployment and Inventory Management Problem (MFDIMP), 
along with computational testings on realistically generated test instances from the Ro-Ro shipping companies. The fleet deployment combined with inventory management in Ro-Ro shipping is rarely dealt with in the literature, but a few studies exist. Chandra et al. $[4,5]$ propose a time-discrete model for the combined MFDIMP. In deep sea shipping where the planning horizons can be long, the time-continuous formulation is preferred as it is impractical to use time discretization with large number of time periods in time-discrete model.

The remainder of this paper is organized as follows: In the next section we give a thorough description of the combined MFDIMP. Section 3 presents the mathematical formulation for the problem with a special focus on the modeling of the inventories at ports. A computational study is performed in Section 4, while concluding remarks are given in the final section.

\section{Problem Description}

We consider a liner shipping company that operates a heterogeneous fleet of ships with different capacities, service speeds and bunker consumption. At the beginning of the planning horizon, all ships in the fleet have unique initial positions, either in a port or somewhere at sea. Moreover, the ships can have different preparation times before they are able to commence new voyages because they must continue their on-going voyages or dry dock (for example, repair) first.

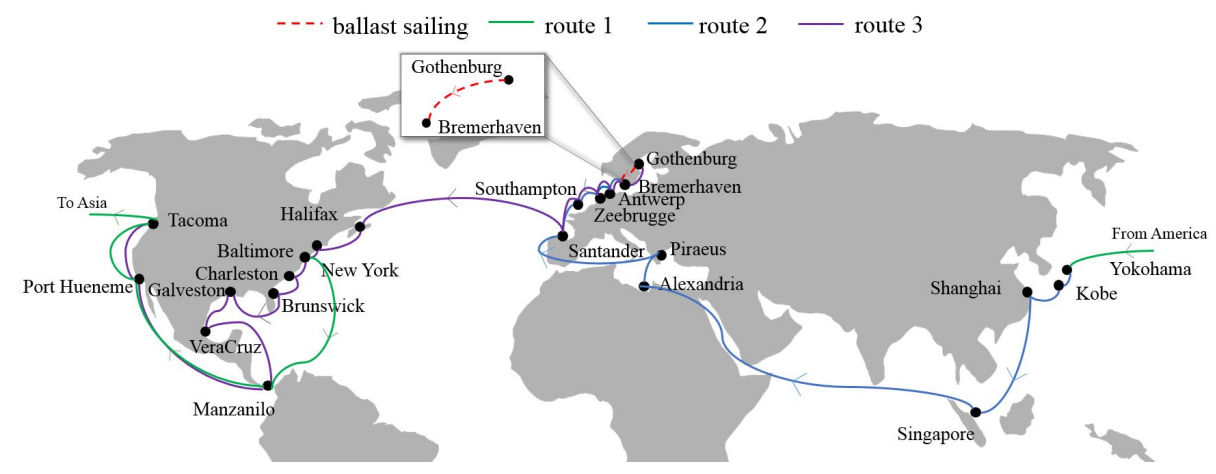

Fig. 1. A three route problem: America to Asia, Asia to Europe, Europe to America, with associated ballast sailings

A route (also called service) is defined as a logistical network used to transport all cargo from their loading ports in one geographical region to unloading ports in another. In general, the shipping company serves several routes. In Fig. 1 , three routes are illustrated by solid lines and the ports are shown as dots. Route 1 sails from America to Asia while route 2 connects four Asian ports with five European ports. Similarly, route 3 starts from Europe and sails to America. To maintain regular service, each route must be serviced according to a given 
frequency, e.g. weekly or bi-weekly. Each sailing along a route is called a voyage. Even though each route should be serviced according to the given frequency, there is some flexibility when each voyage along a route must start, given by a time window. Each voyage (for example voyage number 1 on route 1 ) must be sailed by a ship and is often called a mandatory voyage. After a ship has completed one voyage on a route, it may serve another voyage on the same route or another route or end its service. To start the next voyage, the ship sails without load (i.e. ballast sailing) to the first port of the next voyage. As an example, a ship may first serve a voyage on route 2 from Yokohama in the Asia. This route ends at Gothenburg in the Europe. The ship may then continue to serve a voyage on route 3 from Bremerhaven in the Europe. In this case, there would be a ballast sailing from Gothenburg to Bremerhaven between the two voyages. The ballast sailing between the two routes in Fig. 1 is illustrated by a dotted line. Different contract terms and product types transported along the various routes may restrict which ships can be assigned to voyages on a particular route, regarding capacity and compatibility.

We assume deterministic sailing times for all ships between successive ports along a route. We also consider deterministic sailing times for each ship between the last port of one route and the first port of another route. It is assumed that the time spent at each port is given regardless of quantities loaded/unloaded as the most important part of the processing time at a port is to enter/leave the port and berth. Waiting at the first port of a voyage is allowed, but waiting times at successive ports along the voyage are not allowed. A time window associated with each voyage, defined by an earliest and latest start time, defines within what time interval the service in the first port of the voyage must start.

Servicing a voyage incurs costs such as port and fuel consumption costs, depending on the ship type. It is common in this service that the automobile company has responsibility for the storage of the cargo at both the production and consumption ports. Therefore, the inventory costs can be considered as disregarded. This assumption is consistent with most research on maritime inventory routing, where inventory management is still a nontrivial part of the problem even though inventory costs are not considered.

We assume that the shipping company has an option to charter spot ships at a given cost from the market to service a voyage. The spot ships are assumed to be ready to service any voyage during the whole planning horizon, and they can start any time within its time window.

We use the term product for the same type of product transported along the same route. Each product has given production rates at its associated loading ports and given consumption rates at its associated unloading ports, which is assumed constant during the planning horizon. Each port has a different production and consumption rate for specified products. At a production port of a product, the shipping company must determine how many units to load of each product transported to its corresponding unloading ports. Each ship is able to carry a number of different products at the same time, and the products need to be carried in dedicated compartments because some of these products cannot be 
stored together. Similarly, each port has a storage capacity in terms of the maximum number of product it can hold. The size of spot ships for a given product is assumed as the size of the largest ship in the fleet for that product. Cargoes are rarely transshipped in Ro-Ro shipping, so transshipment is disregarded. The aim of this combined MFDIMP is to determine: (a) the ship routes and schedules (i.e. which ship should perform which voyages and in what sequence), (b) the start time of each voyage, (c) which voyages should be serviced by spot ships, and (d) the number of units of each product to be loaded/unloaded at associated ports during each voyage. The problem is to be solved subject to the following constraints: (a) all voyages are serviced within their given time windows, either by a ship in the fleet or by a spot ship, (b) the aggregate inventory limit of all products in a particular port should not exceed the maximum storage limit, and (c) there is no backlogging of demand for any product in any of the ports. The objective is to minimize total costs, which consist of the sailing costs for ships in the fleet and the time charter costs for spot ships, over a given planning horizon.

\section{Model Formulation}

The number of visits to each route during a given horizon is assumed fixed in Ro-Ro shipping. Thus, we use a time-continuous formulation which considers an ordering of the route visits according to the time of the visit, and introduce an index indicating the visit number to a particular route (i.e., voyage). The ship paths are defined on a network where the nodes correspond to route visits. As far as we know, there exist no studies in the literature which use a similar modeling approach as the one proposed in this paper.

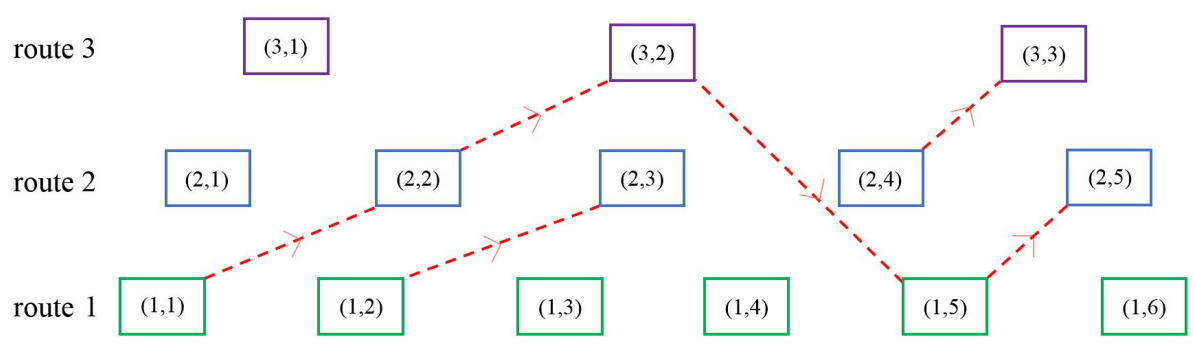

Fig. 2. Illustration of nodes and arcs with three ships: a ship services voyage 1 on route 1 , voyage 2 on route 2 , voyage 2 on route 3 , voyage 5 on route 1 and voyage 5 on route 2 ; a ship services voyage 2 on route 1 , voyage 3 on route 2 ; a ship services voyage 4 on route 2 , voyage 3 on route 3 .

We need to explain how nodes and arcs should be interpreted before we start with the modeling. In this model a node represents a given voyage along a route as illustrated in Fig.2. Thus, the pair denoted by $(i, m)$ corresponds to a node in the model, i.e. voyage number $m$ on route number $i$. Inside each node, 
route 2 voyage $2(2,2)$

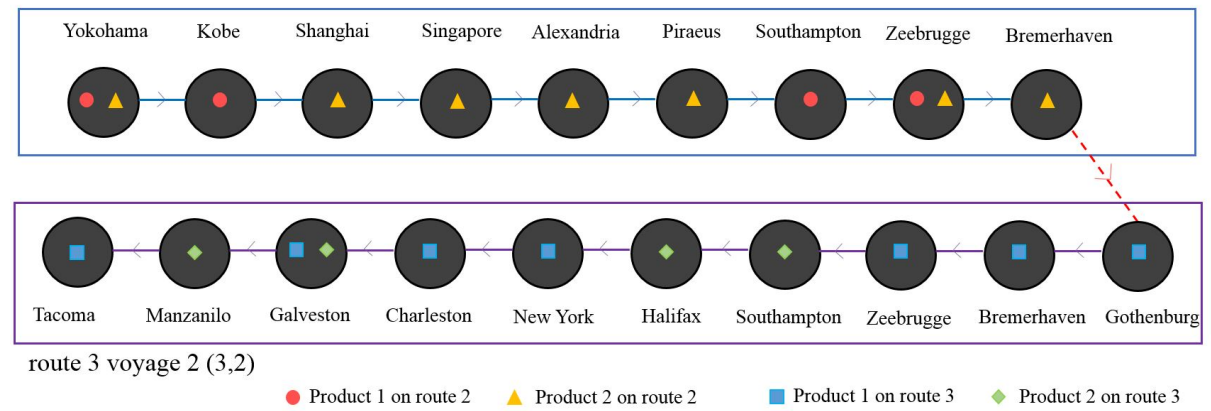

Fig. 3. Illustration of structure inside nodes: set of ports along the two routes. In $(2,2)$, a ship loads product 1 and 2 at Yokohama, sails and loads product 1 at Kobe, sails and loads product 2 at Shanghai and Singapore, sails and unloads product 2 at Alexandria and Piracus, sails and unloads product 1 at Southampton, sails and unloads product 1 and 2 at Zeebrugge, finally arrives at Bremerhaven and unloads product 2. Similarly, a ship carries two types of products from their associated loading ports to unloading ports in $(3,2)$.

all the ports along the route are visited by the ship in sequence as shown in Fig.3. The arc between the nodes is represented by dotted line and denote the ballast sailing. For example in Fig.2 and Fig.3, the arc $\{(2,2)(3,2)\}$ represents the ballast sailing from the last port of voyage number 2 on route 2 to the first port of voyage number 2 on route 3 . For a given route of a ship $v$ in Fig.3, the decision variable $x_{2232 v}$ is a binary variable that is equal to 1 if the ship performs voyages $(2,2)$ and $(3,2)$ in sequence, and 0 otherwise.

Section 3.1 describes the notations used in the model, while the mathematical model for the problem in Section 2 is presented in Section 3.2. Finally, the nonlinear constraints in the model are linearized in Section 3.3.

\subsection{Notation}

The notation used in the model is presented in this section for easy reference.

\section{Sets}

$\mathcal{I} \quad$ set of routes, $i \in \mathcal{I}$

$\mathcal{M} \quad$ set of voyages, $m \in \mathcal{M}$

$\mathcal{N} \quad$ set of nodes, i.e. a voyage $m$ along a route $i,(i, m) \in \mathcal{N}$

$\mathcal{N}_{v} \quad$ set of nodes visited by ship $v, \mathcal{N}_{v} \subseteq \mathcal{N}$

$\mathcal{A}_{v} \quad$ set of $\operatorname{arcs}(i, m)(j, n)$ that can be serviced by ship $v$

$\mathcal{V}^{R} \quad$ set of ships in the fleet, $v \in \mathcal{V}^{R}$

$\mathcal{V}^{A} \quad$ set of spot ships, $v \in \mathcal{V}^{A}$

$\mathcal{V} \quad$ set of ships, $\mathcal{V}^{A} \cup \mathcal{V}^{R}=V$

$\mathcal{P}_{i} \quad$ set of ports along route $i, p \in \mathcal{P}_{i}$

$\mathcal{K}_{i} \quad$ set of products transported along route $i, k \in \mathcal{K}_{i}$ 


\section{Parameters}

$M_{i}$

$P_{i}$

$K_{i}$

$C_{i v}^{O}$

$C_{i j v}$

$C_{i v}^{R}$
$T^{O}$

$T_{i v}^{O}$

$T_{i j v}$

$T_{i p}^{P}$

$T_{i p}^{R}$

$\underline{T}_{i m}$

$\bar{T}_{i m}$

$T$

$S_{i p k}^{O}$

$R_{i p k}$

$I_{i p k}$

$\underline{S}_{i p}$

$\overline{\bar{S}}_{i p}$

$Q_{i k v}$

$Q_{v}^{C}$

\section{Variables}

$x_{i m v}^{O}$

$z_{v}^{O}$

$x_{i m j n v}$

$z_{i m v}$

$w_{i m v}$

$t_{i m}$

the number of voyages along route $i$ during the planning horizon

the number of ports along route $i$

the number of products transported along route $i$

cost for ship $v$ sailing from its initial position to the start position of route $i$

cost of performing voyage along route $i$ and sailing ballast from the last port of route $i$ to the first port of route $j$ with ship $v$ cost of performing voyage along route $i$ with ship $v$

sailing time for ship $v$ from its initial position to the start position of route $i$

sailing time of performing voyage along route $i$ and sailing ballast from the last port of route $i$ to the first port of route $j$ with ship $v$

time spent at port $p$ along route $i$

sailing time on route $i$ from the start position of route $i$ to port $p$ the earliest start time of servicing node $(i, m)$

the latest start time of servicing node $(i, m)$

the length of the planning horizon

the initial inventory level of product $k$ at port $p$ along route $i$ at the beginning of the planning horizon

production rate of product $k$ at port $p$ along route $i$, positive if port $p$ is producing the product, and negative if port $p$ is consuming the product

1 if port $p$ is a loading port, -1 if port $p$ is a unloading port, and 0 otherwise

the minimum inventory level at port $p$ on route $i$

the maximum inventory level at port $p$ on route $i$

the capacity of the compartment of ship $v$ dedicated for product $k$ along route $i$

the total capacity onboard ship $v$

1 if ship $v$ sails directly from its initial position to the start position of node $(i, m), 0$ otherwise

1 if $\operatorname{ship} v$ is not used, 0 otherwise

1 if ship $v$ sails directly from node $(i, m)$ to node $(j, n)$ (ship $v$ sails node $(i, m)$ and then ballast sailing to node $(j, n)$ directly afterwards), 0 otherwise

1 if ship $v$ sails node $(i, m)$ as its last voyage, 0 otherwise

1 if ship $v$ visits node $(i, m), 0$ otherwise

the time at which service starts at node $(i, m)$ 
$s_{i m p k} \quad$ inventory level of product $k$ at port $p$ at the start of service for node $(i, m)$

$s_{i m p k}^{E} \quad$ inventory level of product $k$ at port $p$ at the end of service for node $(i, m)$

$l_{\text {impkv }} \quad$ the amount of product $k$ onboard ship $v$ when leaving port $p$ on node $(i, m)$

$q_{i m p k v} \quad$ the amount of product $k$ loaded/unloaded to/from ship $v$ at port $p$ on arrival of node $(i, m)$

\subsection{Mathematical Model}

Objective Function. The objective function (1) is to minimize the total transportation costs. It consists of the sailing costs for ships in the fleet (i.e. initial ballast sailing costs, voyage costs and ballast sailing costs between successive voyages) and the time charter costs for spot ships.

$$
\begin{aligned}
\text { Minimize } & \sum_{v \in \mathcal{V}} \sum_{(i, m) \in \mathcal{N}_{v}} C_{i v}^{O} x_{i m v}^{O}+\sum_{v \in \mathcal{V}^{R}} \sum_{((i, m),(j, n)) \in \mathcal{A}_{v}} C_{i j v} x_{i m j n v}+ \\
& \sum_{v \in \mathcal{V}} \sum_{(i, m) \in \mathcal{N}_{v}} C_{i v}^{R} z_{i m v}
\end{aligned}
$$

Routing Constraints. Constraints (2) ensure that ship $v$ either departs from its initial position and sails towards node $(i, m)$, i.e. to serve a voyage, or it is not used.

$$
\sum_{(i, m) \in \mathcal{N}_{v}} x_{i m v}^{O}+z_{v}^{O}=1, \quad v \in \mathcal{V}
$$

Constraints (3) and (4) are the flow conservation constraints, ensuring that a ship arriving at a node also leaves that node by either visiting another node or ending its route.

$$
\begin{aligned}
& \sum_{(j, n) \in \mathcal{N}_{v}} x_{j n i m v}+x_{i m v}^{O}-w_{i m v}=0, \quad v \in \mathcal{V},(i, m) \in \mathcal{N}_{v} \\
& w_{i m v}-\sum_{(j, n) \in \mathcal{N}_{v}} x_{i m j n v}-z_{i m v}=0, \quad v \in \mathcal{V},(i, m) \in \mathcal{N}_{v}
\end{aligned}
$$

Constraints (5) ensure that ship $v$ either ends after servicing some node or it is not used.

$$
\sum_{(i, m) \in \mathcal{N}_{v}} z_{i m v}+z_{v}^{O}=1, \quad v \in \mathcal{V}
$$

Voyage Completing Constraints. Constraints (6) ensure that each node is serviced once by either a spot ship or a ship in the fleet.

$$
\sum_{v \in \mathcal{V}} w_{i m v}=1, \quad(i, m) \in \mathcal{N}
$$


Load Management Constraints. Constraints (7) and (8) relate the quantity on board a ship to the quantity (un)loaded from the ship. Constraints (7) ensure that if ship $v$ sails from port $p-1$ to port $p$, then the quantity of product $k$ on board at the departure from port $p$ should be equal to the quantity on board at departure from port $p-1$ plus (or minus) the quantity loaded (or unloaded) from $p$.

$$
\begin{gathered}
w_{i m v}\left(l_{i m(p-1) k v}+I_{i p k} q_{i m p k v}-l_{i m p k v}\right)=0, \\
v \in \mathcal{V},(i, m) \in \mathcal{N}_{v}, p \in \mathcal{P}_{i} \backslash\{1\}, k \in \mathcal{K}_{i}
\end{gathered}
$$

We assume that all ships in the fleet start their voyages empty. Constraints (8) relate the quantity on board with the quantity loaded or unloaded at the start position of node $(i, m)$.

$$
\begin{array}{r}
w_{i m v}\left(I_{i 1 k} q_{i m 1 k v}-l_{i m 1 k v}\right)=0, \\
v \in \mathcal{V},(i, m) \in \mathcal{N}_{v}, k \in \mathcal{K}_{i}
\end{array}
$$

Similarly, all ships in the fleet are assumed ending their voyages empty.

$$
l_{i m P_{i} k v}=0, \quad v \in \mathcal{V},(i, m) \in \mathcal{N}_{v}, k \in \mathcal{K}_{i}
$$

The ship capacity constraints are given by (10) and (11) imposing an upper bound on the quantity on board for a single product and all the products respectively. Constraints (10) also impose an lower bound on the the quantity on board.

$$
\begin{gathered}
0 \leq l_{i m p k v} \leq Q_{i k v} w_{i m v}, \quad v \in \mathcal{V},(i, m) \in \mathcal{N}_{v}, p \in \mathcal{P}_{i}, k \in \mathcal{K}_{i} \\
\sum_{k \in \mathcal{K}_{i}} l_{i m p k v} \leq Q_{v}^{C} w_{i m v}, \quad v \in \mathcal{V},(i, m) \in \mathcal{N}_{v}, p \in \mathcal{P}_{i}
\end{gathered}
$$

Constraints (12) impose lower and upper bounds on the quantity loaded or unloaded.

$$
0 \leq q_{i m p k v} \leq Q_{i k v} w_{i m v}, \quad v \in \mathcal{V},(i, m) \in \mathcal{N}_{v}, p \in \mathcal{P}_{i}, k \in \mathcal{K}_{i}
$$

Time Constraints. Constraints (13) ensure that if ship $v$ travels from its initial position to node $(i, m)$ to start a voyage at time $t$, then the service at node $(i, m)$ can only occur after the ship has arrived.

$$
t_{i m} \geq \sum_{v \in \mathcal{V}} T_{i v}^{O} x_{i m v}^{O}, \quad(i, m) \in \mathcal{N}
$$

Constraints (14) ensure that if ship $v$ sails directly from node $(i, m)$ to node $(j, n)$, then the service at node $(j, n)$ can only start after the start time of service at previous node $(i, m)$ plus the time required to travel from route $i$ to route $j$.

$$
x_{i m j n v}\left(t_{i m}+T_{i j v}-t_{j n}\right) \leq 0, \quad v \in \mathcal{V}^{R},((i, m),(j, n)) \in \mathcal{A}_{v}
$$

Constraints (15) define the time windows for each node.

$$
\underline{T}_{i m} \leq t_{i m} \leq \bar{T}_{i m}, \quad(i, m) \in \mathcal{N}_{v}
$$


Inventory Constraints. The inventory constraints are necessary to ensure that the inventory levels are kept within the corresponding bounds and to link the inventory levels to the (un)loading quantities.

Constraints (16) and (17) define the inventory level upon arrival at each port on route $i$ for the first time. Constraints (17) calculate the inventory level of each product at the first port on route $i$ for the first time the route is visited, i.e. first voyage on the route.

$$
\begin{gathered}
s_{i 1 p k}=S_{i p k}^{O}+R_{i p k}\left(t_{i 1}+T_{i p}^{R}+\sum_{p^{\prime}=1}^{p-1} T_{i p^{\prime}}^{P}\right), \quad(i, 1) \in \mathcal{N}, p \in \mathcal{P}_{i} \backslash\{1\}, k \in \mathcal{K}_{i} \\
s_{i 11 k}=S_{i 1 k}^{O}+R_{i 1 k} t_{i 1}, \quad(i, 1) \in \mathcal{N}, k \in \mathcal{K}_{i}
\end{gathered}
$$

The inventory level when service finishes at any port call $(i, m, p)$ can be calculated from the inventory level upon arrival at the port in the call $(i, m, p)$, adjusted for the loaded/unloaded quantity at the port call and the quantity produced/consumed when ships operates at port as in constraints (18).

$$
\begin{gathered}
s_{i m p k}^{E}=s_{i m p k}-I_{i p k} \sum_{v \in \mathcal{V}} q_{i m p k v}+T_{i p}^{P} R_{i p k}, \\
(i, m) \in \mathcal{N}, p \in \mathcal{P}_{i}, k \in \mathcal{K}_{i}
\end{gathered}
$$

The inventory level upon arrival at any port call $(i, m, p)$ can be calculated from the inventory level when the service finishes at the port in the call $(i, m-1, p)$, adjusted for the quantity produced/consumed in between as in constraints (19).

$$
\begin{aligned}
s_{i m p k}= & s_{i(m-1) p k}^{E}+R_{i p k}\left(t_{i m}-t_{i(m-1)}-T_{i p}^{P}\right), \\
& (i, m) \in \mathcal{N} \backslash\{(i, 1)\}, p \in \mathcal{P}_{i}, k \in \mathcal{K}_{i}
\end{aligned}
$$

The upper and lower bounds on the inventory levels are ensured by constraints (20) - (22). For a loading port, the inventory level increase monotonously before the start of any port operation. Therefore the possible maximum inventory level immediately before any loading port operation cannot exceed the upper bounds on the inventory level. Then the inventory level at the end of any port operation decrease due to the operation at the loading port. Therefore the possible minimum inventory level immediately after any loading port operation cannot less than the lower bounds on the inventory level as in (20). (21) describe the similar requirements for unloading ports.

$$
\begin{aligned}
& \sum_{k \in \mathcal{K}_{i}} s_{i m p k} \leq \bar{S}_{i p}, \sum_{k \in \mathcal{K}_{i}} s_{i m p k}^{E} \geq \underline{S}_{i p}, \quad(i, m) \in \mathcal{N}, p \in \mathcal{P}_{i}^{L} \\
& \sum_{k \in \mathcal{K}_{i}} s_{i m p k} \geq \underline{S}_{i p}, \sum_{k \in \mathcal{K}_{i}} s_{i m p k}^{E} \leq \bar{S}_{i p}, \quad(i, m) \in \mathcal{N}, p \in \mathcal{P}_{i}^{U}
\end{aligned}
$$


Constraints (22) ensure that the inventory level at the end of the planning horizon is within the limits.

$$
\begin{gathered}
\underline{S}_{i p} \leq \sum_{k \in \mathcal{K}_{i}}\left(s_{i m p k}^{E}+R_{i p k}\left(T-t_{i m}-T_{i p}^{R}-\sum_{p^{\prime}=1}^{p} T_{i p^{\prime}}^{P}\right) \leq \bar{S}_{i p}\right. \\
(i, m) \in \mathcal{N} \mid m=M_{i}, p \in \mathcal{P}_{i}
\end{gathered}
$$

Binary and Non-negativity Constraints. Constraints (23) - (25) define the variables as binary. The nonnegativity requirements (26) are given for the variables representing the inventory level.

$$
\begin{gathered}
x_{i m j n v} \in\{0,1\}, \quad v \in \mathcal{V}^{R},((i, m),(j, n)) \in \mathcal{A}_{v} \\
x_{i m v}^{O}, z_{i m v}, w_{i m v} \in\{0,1\}, \quad v \in \mathcal{V},(i, m) \in \mathcal{N}_{v} \\
z_{v}^{O} \in\{0,1\}, \quad v \in \mathcal{V} \\
s_{i m p k}, s_{i m p k}^{E} \geq 0, \quad(i, m) \in \mathcal{N}, p \in \mathcal{P}_{i}, k \in \mathcal{K}_{i}
\end{gathered}
$$

\subsection{Linearization}

Since constraints (7) are non-linear and cannot be solved by a linear solver, we linearize the constraints (7) by replacing them with the following two sets of constraints (27) and (28), which forces $l_{i m(p-1) k v}+I_{i p k} q_{i m p k v}=l_{i m p k v}$ when $w_{i m v}=1$.

$$
\begin{aligned}
& l_{i m(p-1) k v}+I_{i p k} q_{i m p k v}-l_{i m p k v} \leq Q_{i k v}\left(1-w_{i m v}\right) \\
& v \in \mathcal{V},(i, m) \in \mathcal{N}_{v}, p \in \mathcal{P}_{i} \backslash\{1\}, k \in \mathcal{K}_{i} \\
& l_{i m(p-1) k v}+I_{i p k} q_{i m p k v}-l_{i m p k v} \geq Q_{i k v}\left(w_{i m v}-1\right), \\
& v \in \mathcal{V},(i, m) \in \mathcal{N}_{v}, p \in \mathcal{P}_{i} \backslash\{1\}, k \in \mathcal{K}_{i}
\end{aligned}
$$

Similarly, non-linear constraints (8) can be linearized in (29) and (30).

$$
\begin{aligned}
& I_{i 1 k} q_{i m 1 k v}-l_{i m 1 k v} \leq Q_{i k v}\left(1-w_{i m v}\right), \quad v \in \mathcal{V},(i, m) \in \mathcal{N}_{v}, k \in \mathcal{K}_{i} \\
& I_{i 1 k} q_{i m 1 k v}-l_{i m 1 k v} \geq Q_{i k v}\left(w_{i m v}-1\right), \quad v \in \mathcal{V},(i, m) \in \mathcal{N}_{v}, k \in \mathcal{K}_{i}
\end{aligned}
$$

Constraints (14) are linearized as constraints (31), following [2].

$$
\begin{gathered}
t_{i m}-t_{j n}+\max \left\{\bar{T}_{i m}+\sum_{\substack{v \in \mathcal{V}^{R}\\
}} T_{i j v}-\underline{T}_{j n}, 0\right\} \sum_{v \in \mathcal{V}^{R}} x_{i m j n v} \leq \bar{T}_{i m}-\underline{T}_{j n}, \\
((i, m),(j, n)) \in \mathcal{A}
\end{gathered}
$$




\section{Computational Study}

The mathematical model described in Section 3 has been implemented in Mosel using Xpress IVE and solved using Xpress 27.01.02. All computational tests are performed on a Windows 8 computer with an Intel i5 core, 2.2 GHz processor and 8 GB RAM. Section 4.1 describes the test instances used, while the performance of the tests using Xpress is presented and discussed in Section 4.2.

\subsection{Test Instances}

Four sets of test instances have been generated and used in the computational study. The first two sets consists of 6 instances, and the other two sets consists of 8 instances. These test instances are based on reduced versions of a real sized problem faced by a Ro-Ro shipping company, the first set of instances based on the Asia to Europe route, the second and third set of instances based on the three routes shown in Fig. 1, and the fourth set of instances based on five routes. Table 1 summarizes the test instances developed for the computational study.

Table 1. Test instances. For the number of ships, the number on the left denotes the number of ships in the fleet, while the number on the right is the number of available spot ships.

\begin{tabular}{|c|c|c|c|c|c|c|c|}
\hline \multirow{2}{*}{$\frac{\text { Instance }}{9 v 1 r 3 p 2 k 90 d}$} & \multicolumn{7}{|c|}{ Nships Nroutes Nports Nvoyages NportCalls Nproduct Time (days) } \\
\hline & $9 / 0$ & 1 & 3 & 9 & 27 & 2 & 90 \\
\hline $9 v 1 r 6 p 2 k 90 d$ & $9 / 0$ & 1 & 6 & 9 & 54 & 2 & 90 \\
\hline $9 v 1 r 9 p 2 k 90 d$ & $9 / 0$ & 1 & 9 & 9 & 81 & 2 & 90 \\
\hline$\overline{7 v 3 r 9 p 6 k 90 d}$ & $7 / 3$ & 3 & 9 & 27 & 81 & 6 & $\overline{90}$ \\
\hline $10 v 3 r 17 p 6 k 90 d$ & $10 / 3$ & 3 & 17 & 27 & 162 & 6 & 90 \\
\hline $10 v 3 r 28 p 6 k 90 d$ & $10 / 3$ & 3 & 28 & 27 & 225 & 6 & 90 \\
\hline $10 v 3 r 28 p 11 k 90 d$ & $10 / 3$ & 3 & 28 & 27 & 225 & 11 & 90 \\
\hline $10 v 3 r 28 p 11 k 120 d$ & $10 / 3$ & 3 & 28 & 36 & 300 & 11 & 120 \\
\hline $10 v 3 r 28 p 11 k 150 d$ & $10 / 3$ & 3 & 28 & 45 & 375 & 11 & 150 \\
\hline $10 v 3 r 28 p 11 k 180 d$ & $10 / 3$ & 3 & 28 & 54 & 450 & 11 & 180 \\
\hline $18 v 5 r 47 p 17 k 90 d$ & $18 / 3$ & 5 & 47 & 40 & 341 & 17 & 90 \\
\hline $18 v 5 r 47 p 17 k 120 d$ & $18 / 3$ & 5 & 47 & 53 & 451 & 17 & 120 \\
\hline $18 v 5 r 47 p 17 k 150 d$ & $18 / 3$ & 5 & 47 & 67 & 572 & 17 & 150 \\
\hline $18 v 5 r 47 p 17 k 180 d$ & $18 / 3$ & 5 & 47 & 80 & 682 & 17 & 180 \\
\hline
\end{tabular}

The information on routes and ports with respective sailing distances and corresponding costs and sailing times are based on real data. Two ship types are considered, large and small, with capacities of approximately 7100 and 5800 Car Equivalent Units (CEUs), respectively. The cost associated with assigning a voyage to a spot ship is assumed almost three times the variable (i.e. fuel and port) cost of serving the voyage with a large ship from the company's own fleet. 
The cost of serving the voyage with a small ship is set to be $20 \%$ lower than the cost of serving the voyage with a large ship from the company's own fleet.

The number of voyages on each route during the planning horizon, time windows for starting the voyages, products produced/consumed on a route and production/consumption rate of respective products at each port are estimated by the authors. Inventory management is taken into consideration at both the production and the consumption ports of each product. The number of products to be transported along each route ranges between 2 and 5 . The daily production or consumption rates of a product at the respective ports are estimated as a random number between 10 and 100 units of the product.

The typical planning horizon varies from months to a year. Here we consider planning horizons of 90, 120, 150 and 180 days, respectively. The instances are named according to the number of ships in the fleet $(v)$, routes $(r)$, ports $(p)$, products $(k)$ and length of planning horizon $(d)$, for example instance $9 v 1 r 3 p 2 k 90 d$ represent test instance with nine ships, one route, three ports, two products and planning horizon of 90 days. For each test instance, the number of ships, routes, ports, voyages, port calls, products and length of planning horizon (in days) are given.

\subsection{Computational Performance}

Table 2. Computational results from the MIP model for the test instances.

\begin{tabular}{lrrrrrr}
\hline Instance & \multicolumn{2}{c}{ ProblemSize } & \multicolumn{3}{c}{ Best Sol.Value Gap(\%) Spot Ship Time(s) } \\
& \# Constraint \# Variable & $(\mathrm{M} \$)$ & & & \\
\hline $9 v 1 r 3 p 2 k 90 d$ & 2831 & 1341 & 5.09 & 0.00 & 0 & 0.5 \\
$9 v 1 r 6 p 2 k 90 d$ & 5240 & 2421 & 5.29 & 0.00 & 0 & 1.0 \\
$9 v 1 r 9 p 2 k 90 d$ & 7649 & 3501 & 5.49 & 0.00 & 0 & 1.9 \\
\hline $7 v 3 r 9 p 6 k 90 d$ & 10358 & 10261 & 22.21 & 0.00 & 3 & 12.6 \\
$10 v 3 r 17 p 6 k 90 d$ & 21516 & 17098 & 24.51 & 0.00 & 3 & 12.4 \\
$10 v 3 r 28 p 6 k 90 d$ & 30913 & 21298 & 30.70 & 0.00 & 3 & 39.3 \\
\hline $10 v 3 r 28 p 11 k 90 d$ & 52642 & 32302 & 30.70 & 0.00 & 3 & 73.9 \\
$10 v 3 r 28 p 11 k 120 d$ & 70695 & 46523 & 39.09 & 0.00 & 3 & 165.6 \\
$10 v 3 r 28 p 11 k 150 d$ & 89014 & 62473 & 42.98 & 0.00 & 3 & 1599.3 \\
$10 v 3 r 28 p 11 k 180 d$ & 107599 & 80152 & 50.21 & 0.00 & 3 & 1636.2 \\
\hline $18 v 5 r 47 p 17 k 90 d$ & 118536 & 84406 & 40.09 & 0.00 & 2 & 40.2 \\
$18 v 5 r 47 p 17 k 120 d$ & 158126 & 124587 & 52.27 & 0.00 & 3 & 193.9 \\
$18 v 5 r 47 p 17 k 150 d$ & 201789 & 175209 & 68.05 & 0.50 & 3 & 3600.0 \\
$18 v 5 r 47 p 17 k 180 d$ & 242625 & 228473 & 79.70 & 1.31 & 3 & 3600.0 \\
\hline
\end{tabular}

The computational results for solving the MIP model are presented in Table 2. The size of the model for each of the instances is presented in terms of number of constraints and variables. Moreover, Table 2 gives details related to the best MIP solution value (in M\$) obtained, and the optimality gap (in \%), Gap = 
$\frac{\text { BestIntegerSolutionValue-BestBound }}{\text { BestIntegerSolutionValue }} \times 100$, is reported if the model could not be solved to optimality within the time limit which is set to one hour. The actual number of spot ships used is also reported.

The MIP model could be solved to optimality for most small instances within reasonable times except for the two largest ones, $18 v 5 r 47 p 17 k 150 d$ and $18 v 5 r 47 p 17 k 180 d$, for which the feasible integer solutions are found and the gaps are reported are $0.5 \%$ and $1.3 \%$, respectively.

Even for the two largest instances, $18 v 5 r 47 p 17 k 150 d$ and $18 v 5 r 47 p 17 k 180 d$, good solutions are obtained within the time limit. It is however clear that the computational time increases rapidly with the increase of the number of ships, routes, ports and products. The real problem that can involve up to 19 routes and 60 ships, as well as a larger number of products would result in much longer computational time. Moreover, the computational results also show that the size of the instances as well as the computational time increases dramatically with the increased length of the planning horizon. For budgeting reasons, some Ro-Ro shipping companies could be interested in solving problems with up to one year planning horizons, which would be impossible using only a commercial solver (like Xpress).

\section{Concluding Remarks}

In this paper, we have presented a planning problem faced by Ro-Ro shipping companies providing integrated logistic services to its customers. We have proposed a new mathematical model, combining inventory management at the ports with the planning of ship routes. This planning problem is called a combined Maritime Fleet Deployment and Inventory Management Problem (MFDIMP).

Test instances are created based on reduced version of a real Ro-Ro shipping company. Out of the 14 test instances, only the two largest instances with 18 ships, 5 routes and planning horizons of 150 and 180 days could not be solved to optimality. The computational results suggest that the time-continuous formulation is likely to perform better than the time-discrete model in [4], particularly for instances with a long planning horizon.

It should be emphasized that even though the results are promising for our test instances, the combined MFDIMP is a very complex problem and instances of realistic size with more ships, routes and longer planning horizon could be even harder or impossible to solve within reasonable computational times by only using a commercial mixed-integer programming solver. Therefore, there are many possible directions for future research. In order to find optimal solutions within reasonable computational times, it would be interesting to study exact solution techniques, for example decomposition approaches. Moreover, the possibility of tightening the formulation and including suitable valid inequalities for the inventory management part of the problem could also be explored. In addition, advanced heuristics could also be developed to obtain near optimal solutions for large realistic instances of the problem. 


\section{References}

1. Agra, A., Christiansen, M., Delgado, A.: Discrete time and continuous time formulations for a short sea inventory routing problem. Optimization and Engineering 18(1), 269-297 (2017)

2. Agra, A., Christiansen, M., Figueiredo, R., Hvattum, L.M., Poss, M., Requejo, C.: The robust vehicle routing problem with time windows. Computers \& Operations Research 40(3), 856-866 (2013)

3. Andersson, H., Fagerholt, K., Hobbesland, K.: Integrated maritime fleet deployment and speed optimization: Case study from roro shipping. Computers \& Operations Research 55, 233-240 (2015)

4. Chandra, S., Christiansen, M., Fagerholt, K.: Combined fleet deployment and inventory management in roll-on/roll-off shipping. Transportation Research Part E: Logistics and Transportation Review 92, 43-55 (2016)

5. Chandra, S., Fagerholt, K., Christiansen, M.: Maritime fleet deployment in ro-ro shipping under inventory constraints. Procedia - Social and Behavioral Sciences 189, 362-375 (2015)

6. Christiansen, M., Fagerholt, K.: Ship routing and scheduling in industrial and tramp shipping. In: Toth, P., Vigo, D. (eds.) Vehicle Routing: Problems, Methods, and Applications, chap. 13, pp. 381-408. Society for Industrial and Applied Mathematics Philadelphia (2014)

7. Christiansen, M., Fagerholt, K., Nygreen, B., Ronen, D.: Ship routing and scheduling in the new millennium. European Journal of Operational Research 228(3), 467-483 (2013)

8. Fagerholt, K., Johnsen, T.A.V., Lindstad, H.: Fleet deployment in liner shipping: a case study. Maritime Policy \& Management 36(5), 397-409 (2009)

9. Hemmati, A., Hvattum, L.M., Christiansen, M., Laporte, G.: An iterative twophase hybrid matheuristic for a multi-product short sea inventory-routing problem. European Journal of Operational Research 252(3), 775-788 (2016)

10. Jaramillo, D.I., Perakis, A.N.: Fleet deployment optimization for liner shipping part 2. implementation and results. Maritime Policy \& Management 18(4), 235$262(1991)$

11. Papageorgiou, D.J., Nemhauser, G.L., Sokol, J., Cheon, M.S., Keha, A.B.: MIRPLib - A library of maritime inventory routing problem instances: Survey, core model, and benchmark results. European Journal of Operational Research 235(2), 350-366 (2014)

12. Perakis, A.N., Jaramillo, D.I.: Fleet deployment optimization for liner shipping part 1. background, problem formulation and solution approaches. Maritime Policy \& Management 18(3), 183-200 (1991)

13. Powell, B.J., Perkins, A.N.: Fleet deployment optimization for liner shipping: an integer programming model. Maritime Policy \& Management 24(2), 183-192 (1997)

14. Savelsbergh, M., Song, J.H.: An optimization algorithm for the inventory routing problem with continuous moves. Computers \& Operations Research 35(7), 2266$2282(2008)$

15. Siswanto, N., Essam, D., Sarker, R.: Solving the ship inventory routing and scheduling problem with undedicated compartments. Computers \& Industrial Engineering 61(2), 289-299 (2011)

16. Song, J.H., Furman, K.C.: A maritime inventory routing problem: Practical approach. Computers \& Operations Research 40(3), 657-665 (2013)

17. Stålhane, M., Andersson, H., Christiansen, M., Fagerholt, K.: Vendor managed inventory in tramp shipping. Omega 47, 60-72 (2014) 